\title{
Editorial
}

\section{Adjuvant Immunotherapy after Curative Treatment for Hepatocellular Carcinoma}

\author{
Masatoshi Kudo \\ Department of Gastroenterology and Hepatology, Kindai University Faculty of Medicine, Osaka-Sayama, Japan
}

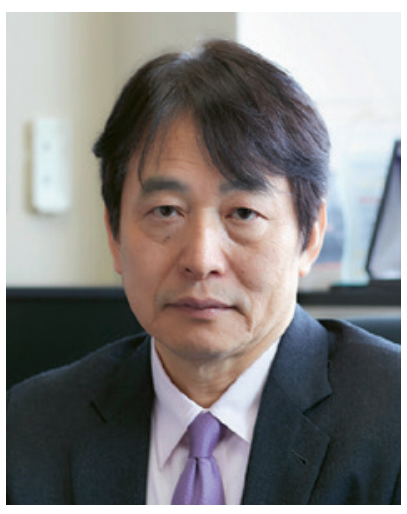

Prof. M. Kudo

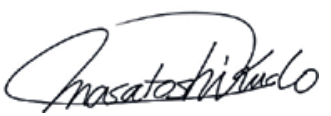

Editor Liver Cancer

\section{Keywords}

Hepatocellular carcinoma · Nivolumab · Adjuvant immunotherapy $\cdot$ Anti-VEGF antibody

\section{Introduction}

Hepatectomy and radiofrequency ablation (RFA) have been established as curative therapies for hepatocellular carcinoma (HCC); however, HCC still has a high recurrence rate after these treatments. A study of HCC pathology has shown that microscopic intrahepatic metastasis occurs in about $10 \%$ of tumors $\leq 2 \mathrm{~cm}$, and microvascular

karger@karger.com www.karger.com/lic

Karger"

BOPEN ACCESS
C 2021 The Author(s).

Published by S. Karger AG, Basel

This is an Open Access article licensed under the Creative Commons Attribution-NonCommercial-4.0 International License (CC BY-NC) (http://www.karger.com/Services/OpenAccessLicense), applicable to the online version of the article only. Usage and distribution for commercial purposes requires written permission. invasion occurs in about $27 \%$ of these tumors [1]. Thus, even small HCCs $(\leq 2 \mathrm{~cm})$ have a risk of recurrence. Moreover, intrahepatic metastasis is a major problem for tumors $>2 \mathrm{~cm}$ because increasing tumor size past this cutoff is correlated with an increased risk of intrahepatic metastasis and microvascular invasion [2]. One reason why the prognosis of HCC is so poor is that patients experience repeated recurrences even after curative treatment, and repeated treatments with RFA, hepatectomy, and transarterial chemoembolization deteriorate liver function in many patients, ultimately resulting in death from liver failure or from tumors that are not amenable to treatment with systemic therapy. This suggests that preventing recurrence of HCC after curative treatment could dramatically improve the prognosis. Several studies on the prevention of recurrence have been conducted to date, but all have failed [3-5]. Consequently, there currently remains an unmet need for research on adjuvant therapy after curative treatment for HCC.

The most prominent clinical studies of adjuvant therapy for recurrence prevention conducted to date investigated vitamin K [3], retinoids (the NIK-333 study) [4], and sorafenib (the STORM trial) [5]. However, the 1-year recurrence rates were high $(25 \%, 34 \%$, and $42 \%$, respectively) in the control arms of all these studies. Another

Masatoshi Kudo is the Editor-in-Chief of Liver Cancer. 


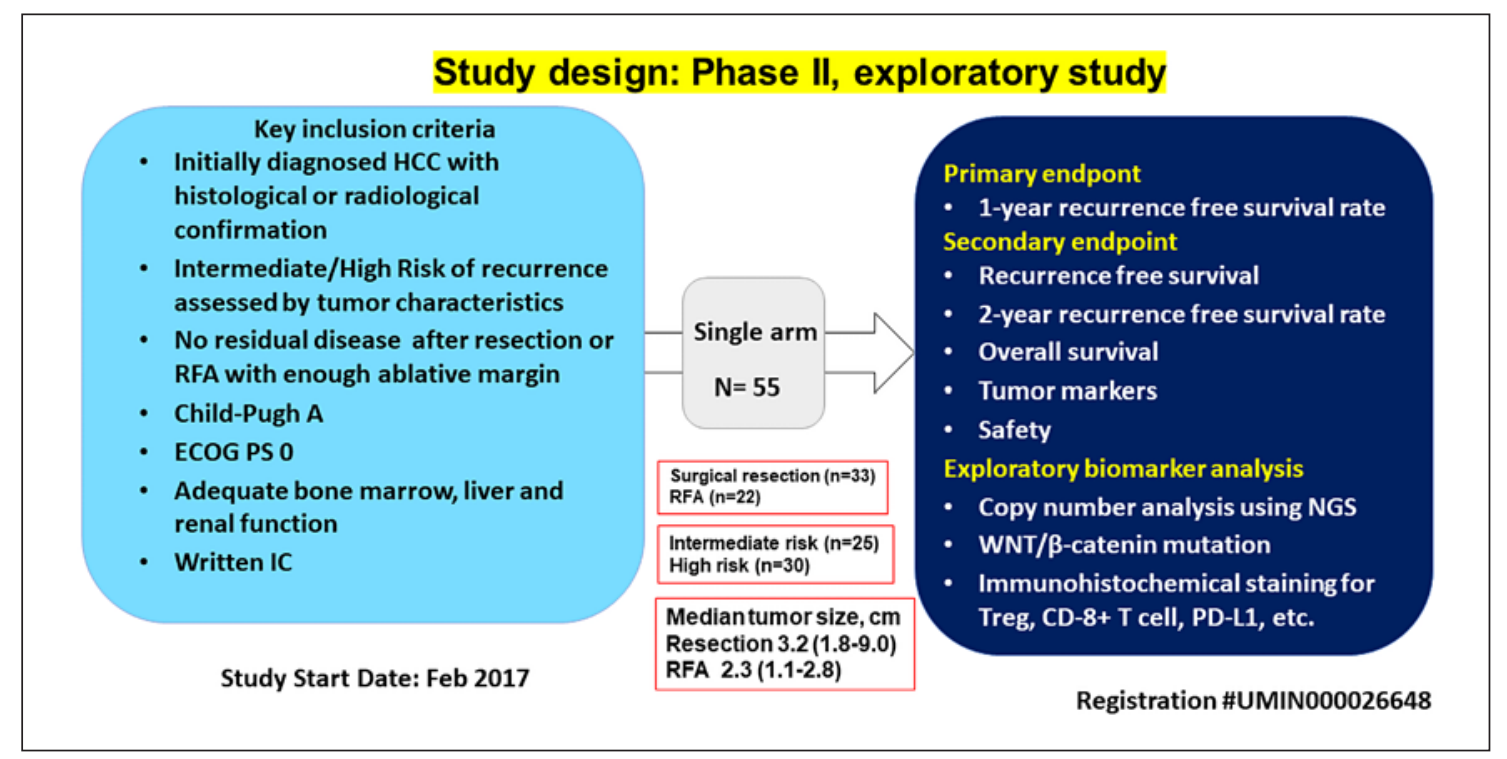

Fig. 1. Study design of NIVOLVE. NIVOLVE is a phase 2 prospective multicenter single-arm trial of adjuvant nivolumab treatment for hepatocellular carcinoma after surgical resection or radiofrequency ablation.

study showed a 5-year recurrence rate of about $80 \%$ after curative treatment for HCC.

Recurrence after curative treatment can occur early or late. Early recurrence is primarily associated with intrahepatic metastasis, whereas late recurrence is often multicentric in origin [6].

\section{Rationale for the Use of Immune Checkpoint Inhibitors (ICls) to Prevent Recurrence of HCC}

Hepatectomy and local ablation using RFA or microwave ablation are known to increase immunogenicity [7]. With ablation specifically, treatment causes the tumor to release tumor-associated antigens, and antigen-presenting cells present these antigens to CD8+ T cells. Upon recognizing these antigens, the $\mathrm{CD} 8+\mathrm{T}$ cells become activated and reach cancer sites such as microsatellite lesions, where they recognize and attack the cancer cells. The rationale for ICI therapy for preventing recurrence is that using an anti-programmed death (PD)-1/PD-ligand (L)1 antibody to prevent immune escape via the PD-1/ PD-L1 pathway could induce necrosis of residual microsatellite lesions in the liver. Duffy et al. [8] have already validated this rationale by showing that ICI therapy after ablation or transarterial chemoembolization for some advanced HCC nodules induced infiltration of CD8+ T cells into the nontreated nodules.

\section{Studies of ICI Monotherapy for Prevention of Recurrence}

Table 1 shows ongoing studies of adjuvant ICI monotherapy and combination immunotherapy with bevacizumab for HCC after curative therapy. There are 2 phase III studies of ICI monotherapy, the CheckMate 9DX study with nivolumab and the KEYNOTE-937 study with pembrolizumab. Results of a single-arm phase II study of nivolumab (NIVOLVE trial; registration number, UMIN000026648) were presented at the annual meeting of the American Society of Clinical Oncology (ASCO) in 2021 (Fig. 1) [9]. The 1-year recurrence rate, which was the primary endpoint, was $23.3 \%$, which was lower than the rate of $42 \%$ achieved in the STORM trial. However, exploratory biomarker analysis in patients who underwent hepatectomy showed that copy number gains in WNT/ $\beta$-catenin-related genes (APC, CTNNB1, TCF7L1, and TCF7L2), $\beta$-catenin activating mutations, low PD-L1 expression, a low CD8+ tumor-infiltrating lymphocyte count, and FOXP3 positivity on immunohistochemical staining were significantly correlated with a high recurrence risk. This suggests that intrahepatic HCC metastases that occur postoperatively have the same immunosuppressive tumor microenvironment and gene mutations associated with immunotherapy resistance (WNT/ $\beta$-catenin mutations) as advanced HCC $[10,11]$. 


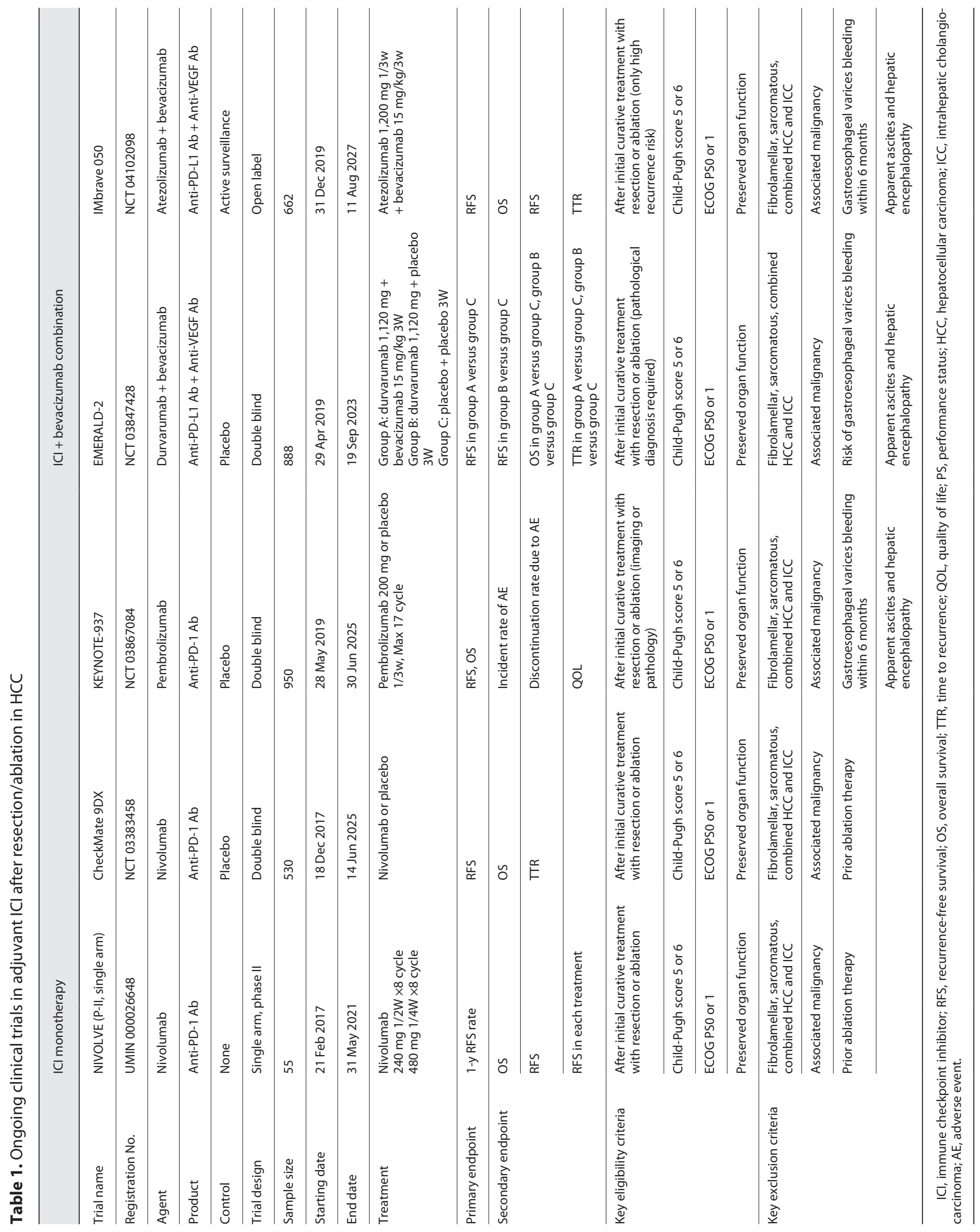


Table 2. Immune mechanisms implicated in hepatocellular carcinoma recurrence following curative resection

\begin{tabular}{ll}
\hline Immune cell/immune checkpoint & Association with recurrence/survival \\
\hline $\begin{array}{ll}\text { I. Tumor infiltration of immune cell } \\
\text { Immune cells }\end{array}$ & $\uparrow$ CD3+ T cells, CD8+ T cells infiltration associated with $\downarrow$ recurrence \\
NKT cells, IFN- $\gamma$ & NKT cells, IFN- $\gamma$ independently predicts recurrence and survival \\
Immune gene signatures & Multiple immune gene signatures associated with the prognosis of \\
& resected HCC \\
II. Accumulation of immunosuppressive cells & $\uparrow$ Treg associated with $\downarrow$ OS and RFS \\
Treg & $\uparrow$ MDSCS correlated with early recurrence after resection \\
MDSC & $\uparrow$ PD-L1+ MDSCs correlated with early recurrence after resection \\
& $\uparrow$ Peritumoral macrophages associated with recurrence and poor survival \\
Macrophages & after hepatectomy \\
III. Upregulation of negative immune checkpoints & $\uparrow$ PD-L1 associated with high-risk factors for recurrence \\
PD-L1 &
\end{tabular}

Modified from Ref. [12].

Previous studies on the involvement of tumor-associated immune factors in HCC recurrence after curative treatment have shown that infiltration by immune cells, particularly CD8+ T cells and natural killer T cells, as well as the production of interferon- $\gamma$, is associated with a lower rate of recurrence $[9,12]$. Studies have also shown that infiltration by suppressive cells such as regulatory $\mathrm{T}$ cells (Tregs) and myeloid-derived suppressor cells is associated with a higher recurrence rate and a worse prognosis [12], and that expression of the immune checkpoint $\mathrm{PD}-\mathrm{L} 1$ is also involved in postoperative recurrence [12] (Table 2).

The NIVOLVE trial reproduced these findings on tumor-associated immune factors and the efficacy of ICIs in preventing recurrence after curative treatment. This trial demonstrated not only that nivolumab therapy works in tumors that express PD-L1 by blocking the PD-1/PD-L1 pathway but also that tumor infiltration by $\mathrm{CD} 8+\mathrm{T}$ cells and the expression of PD-L1 are well correlated with the efficacy of nivolumab in preventing recurrence [9]. In addition, the high recurrence rate among tumors infiltrated by Tregs also indicated that even microsatellite lesions of primary tumors treated by resection or ablation have the same immunosuppressive tumor microenvironment as the original treated HCC, and thus show only a limited response to ICI monotherapy. Finally, the NIVOLVE trial demonstrated that ICI monotherapy is ineffective in preventing recurrence because tumor infiltration by $\mathrm{CD} 8+\mathrm{T}$ cells is suppressed when the $\beta$-catenin signaling pathway is activated and Tregs are infiltrated in the tumor [9].

\section{Rationale and Phase III Studies of ICls in Combination with Anti-Vascular Endothelial Growth Factor Antibody Therapy to Prevent Recurrence of HCC}

As was shown in the NIVOLVE trial, ICI monotherapy is not very effective for preventing recurrence in HCC with an immune suppressive microenvironment because microsatellite lesions of original tumors removed by curative hepatectomy have the same immune microenvironment as the primary HCC. Therefore, it is only effective for the roughly $20 \%$ of tumors that are classified into the immune-active class according to the classification system developed by Sia et al. [13]. The NIVOLVE trial also demonstrated that ICI monotherapy is less effective for the roughly $10 \%$ of tumors that are classified into the immune-exhausted class, which have an immunosuppressive microenvironment. As was demonstrated in Arm F of a phase Ib study of atezolizumab plus bevacizumab versus atezolizumab monotherapy [14], combination therapy with the anti-VEGF antibody bevacizumab is effective because it activates priming by inducing maturation of dendritic cells, increases tumor infiltration by immune cells, and improves immune responsiveness by changing the immune microenvironment from suppressive to permissive. ICI plus anti-VEGF antibody therapy was already shown to be effective in advanced HCC in the IMbrave 150 study [15]. These findings reaffirm the need to use an anti-VEGF antibody to improve the immune microenvironment from immune suppressive to immune permissive, even for adjuvant therapy [12]. Combination therapy with an anti-VEGF antibody is currently being investi- 
gated in 2 phase III studies, the EMERALD-2 study with durvalumab plus bevacizumab and the IMbrave050 study with atezolizumab plus bevacizumab [12] (Table 1).

\section{Conclusion}

The NIVOLVE trial showed that adjuvant ICI monotherapy after curative treatment is effective in CD8+ Tcell-infiltrated HCCs. However, it was ineffective for preventing recurrence in HCC with Treg infiltration or $\beta$-catenin activation, just as it is for advanced HCC. Although no conclusion has been reached as to whether ICI plus anti-VEGF antibody therapy is effective against HCC with $\beta$-catenin activating mutations, it appears to show greater promise than ICI monotherapy for preventing the recurrence of $\mathrm{HCC}$ with an immunosuppressive tumor microenvironment.

In any case, we must wait for the results of the 4 ongoing phase III studies. Because the immune-based classification system was originally based on analysis of resected HCC, the concepts of $\beta$-catenin activation and the immune-exhausted and immune-active phenotypes are already well known. We can say that an ICI plus anti-VEGF antibody treatment would be the more reasonable choice of treatment than ICI monotherapy in the adjuvant setting after curative treatment.
The same is also true for advanced HCC: ICI monotherapy was found to have clinical activity in phase I/II studies, but was not found to be effective in phase III studies. However, because these studies used overall survival as the primary endpoint, the negative results may be attributed to long post-progression survival due to posttrial treatment. In that sense, there is a good chance that even ICI monotherapy may prove suppressive effect of recurrence in placebo-controlled trials in the adjuvant setting because recurrence-free survival is the primary endpoint for these trials.

\section{Conflict of Interest Statement}

The author reports the following: lecture: Eisai, Bayer, MSD, BMS, EA Pharma, Eli Lilly, and Chugai; grants: Eisai, Takeda, Otsuka, Taiho, EA Pharma, Gilead Sciences, AbbVie, Sumitomo Dainippon Pharma, Chugai, and Ono Pharma; advisory consulting: Eisai, Ono, MSD, BMS, and Roche. The author is the Editor-inChief of Liver Cancer.

\section{Funding Sources}

None.

\section{References}

1 Nakashima O, Sugihara S, Kage M, Kojiro M. Pathomorphologic characteristics of small hepatocellular carcinoma: a special reference to small hepatocellular carcinoma with indistinct margins. Hepatology. 1995; 22:101-5.

2 Kudo M, Izumi N, Kokudo N, Sakamoto M, Shiina S, Takayama T, et al. Report of the 21st Nationwide Follow-up survey of primary liver cancer in Japan (2010-2011). Hepatol Res. 2021; 51:355-405.

3 Yoshida H, Shiratori Y, Kudo M, Shiina S, Mizuta T, Kojiro M, et al. Effect of vitamin K2 on the recurrence of hepatocellular carcinoma. Hepatology. 2011;54:532-40.

4 Okita K, Izumi N, Matsui O, Tanaka K, Kaneko $\mathrm{S}$, Moriwaki $\mathrm{H}$, et al. Peretinoin after curative therapy of hepatitis $\mathrm{C}$-related hepatocellular carcinoma: a randomized double-blind placebocontrolled study. J Gastroenterol. 2015;50:191202.

5 Bruix J, Takayama T, Mazzaferro V, Chau GY, Yang J, Kudo M, et al. Adjuvant sorafenib for hepatocellular carcinoma after resection or ablation (STORM): a phase 3, randomised, doubleblind, placebo-controlled trial. Lancet Oncol. 2015;16:1344-54.

Adjuvant Immunotherapy after Curative Treatment for HCC
6 Imamura H, Matsuyama Y, Tanaka E, Ohkubo T, Hasegawa K, Miyagawa S, et al. Risk factors contributing to early and late phase intrahepatic recurrence of hepatocellular carcinoma after hepatectomy. J Hepatol. 2003;38:200-7.

7 Mizukoshi E, Yamashita T, Arai K, Sunagozaka $\mathrm{H}$, Ueda T, Arihara F, et al. Enhancement of tumor-associated antigen-specific $\mathrm{T}$ cell responses by radiofrequency ablation of hepatocellular carcinoma. Hepatology. 2013;57:1448-57.

8 Duffy AG, Ulahannan SV, Makorova-Rusher O, Rahma O, Wedemeyer H, Pratt D, et al. Tremelimumab in combination with ablation in patients with advanced hepatocellular carcinoma. Hepatol. 2017;66:545-51.

9 Kudo M, Ueshima K, Nakahira S, Nishida N, Ida $\mathrm{H}$, Minami $\mathrm{Y}$, et al. Adjuvant nivolumab for hepatocellular carcinoma (HCC) after surgical resection (SR) or radiofrequency ablation (RFA) (NIVOLVE): a phase 2 prospective multicenter single-arm trial and exploratory biomarker anlysis 2021. ASCO Annual Meeting. 2021. Abstr \#4070.

10 Harding JJ, Nandakumar S, Armenia J, Khalil DN, Albano M, Ly M, et al. Prospective genotyping of hepatocellular carcinoma: clinical implications of next-generation sequencing for matching patients to targeted and immune therapies. Clin Cancer Res. 2019;25:2116-26.

11 Morita M, Nishida N, Sakai K, Aoki T, Chishina $\mathrm{H}$, Takita $\mathrm{M}$, et al. Immunological microenvironment predicts the survival of the patients with hepatocellular carcinoma treated with antiPD-1 antibody. Liver Cancer. 2021;10:380-93.

12 Hack SP, Spahn J, Chen M, Cheng AL, Kaseb A, Kudo M, et al. IMbrave 050: a Phase III trial of atezolizumab plus bevacizumab in high-risk hepatocellular carcinoma after curative resection or ablation. Future Oncol. 2020;16:975-89.

13 Sia D, Jiao Y, Martinez-Quetglas I, Kuchuk O, Villacorta-Martin C, Castro de Moura M, et al. Identification of an immune-specific class of hepatocellular carcinoma, based on molecular features. Gastroenterology. 2017;153:812-26.

14 Lee M, Ryoo B-Y, Hsu C-H, Numata K, Stein S, Verret W, et al. Randomised efficacy and safety results for atezolizumab (Atezo) + bevacizumab (Bev) in patients (pts) with previously untreated, unresectable hepatocellular carcinoma (HCC). Ann Oncol. 2019;30:v875.

15 Finn RS, Qin S, Ikeda M, Galle PR, Ducreux M, Kim TY, et al. Atezolizumab plus bevacizumab in unresectable hepatocellular carcinoma. N Engl J Med. 2020;382:1894-905.

Treatment for HCC 